\title{
Influence of Solvents on the Polarization Distribution in PVDF
}

\author{
B. Dehlen, G. Eberle and W. Eisenmenger, \\ Physikalisches Institut, Universität Stuttgart, Germany
}

\begin{abstract}
It is well known that poling of PVDF in high electric fields ( $\sim$ $100 \mathrm{MV} / \mathrm{m}$ ) leads to remanent polarization and a strong piezoelectric effect. The remanent polarization is stable for years at room temperature. We report on polarization reductions if poled films are immersed in polar solvents like acetone, propanol, ethanol, and methanol for a few minutes. Also immersion in nonpolar solvents like hexane or carbon tetrachloride for some hours results in a small reduction of polarization. The amount of the reduction depends on the dipole moment of the solvent molecules, the length of the influence of the solvent, and the poling parameters. We also observe an influence on the remanent polarization if the films have been immersed in a solvent for some minutes before poling. We find inhomogenious polarization distributions in these films different to distributions in untreated films before poling. In the case of a polar solvent pretreatment, the developement of polarization profiles with several maxima is observed. These phenomena are discussed in terms of the interaction of solvent molecules with trapped charges stabilizing the polarization.
\end{abstract}

\section{Introduction}

PVDF films are resistant to most anorganic and organic acids, to oxidation media, aliphatic and aromatic hydrocarbons, to solvents containing halogen and to alcohols. Therefore PVDF is mainly used to protect other materials from these substances [5]. But on the other hand it is well known that polar solvents like acetone and alcohols are absorbed by PVDF. If poled films are immersed into these solvents, the influence on the remanent polarization which is stable without any treatment at room temperature for many years is unknown but of great technical interest because in most applications the surfaces of the polymer film must be very clean and therefore the films are treated with solvents. To investigate the influence of polar solvents we immersed poled PVDF films in acetone and some alcohols for different length of time. The remanent polarization remaining after this solvent treatment has been compared with the polarization after poling the sample. We expect that especially polar solvents 
will have an influence on the polarization by possibly shielding internal fields especially if polar crystallites are not completely neutralised. With increasing dipole moment of the solvent we expect a stronger influence. In addition to this the solubility of polar solvents in PVDF iicreases with dipole moment and reduced molecular size. Therefore we expect that acetone has a greater influence on the remanent polarization as alcohols. On the other hand non polar solvents like hexane and $\mathrm{CCl}_{4}$ presumably may not influence the remanent polarization in poled PVDF. To check this we immerse polarized PVDF films into nonpolar solvents, too.

Since the polarization distribution in PVDF is determined by injected and trapped charges [2] [3] [6] the presence of polar solvents in unpoled films will also influence the polarization built up whereas the influence of non polar solvents will be much smaller. Thus we also investigated the influence of polar and nonpolar solvents by poling PVDF films exposed to these solvents prior to poling.

\section{Experimental}

Samples used were biaxial stretched ca. $35 \mu \mathrm{m}$ thick PVDF films with $70 \%$ $\beta$ content. First the samples were poled at room temperature $(E=220 \mathrm{MV} / \mathrm{m}$, $\mathrm{t}=5 \mathrm{~min}$ or $\mathrm{E}=60 \mathrm{MV} / \mathrm{m}, \mathrm{t}=120 \mathrm{~min})$. The polarization distribution after the poling process was measured with the PPS method [2] [4]. The spatial resolution of this method is about $2 \mu \mathrm{m}$. Afterwards the samples were immersed in different solvents for different times. We used the polar solvents acetone, propanol, ethanol and methanol and the non polar solvents hexane and $\mathrm{CCl}_{4}$. After the exposition to the solvents the polarization distribution in the sample was measured again and compared with the distribution before the samples were immersed in the solvents.

In a second series the PVDF samples were exposed to the solvents for different times bevore the poling process. Then the samples were poled (poling parameters as mentioned above) before evaporation of the solvents out of the samples took place. The polarization distribution was measured again with the PPS method and compared to the distribution in samples poled under the same conditions but without the presence of solvents.

\section{Results}

\subsection{Solvent Contact After Poling}

\subsubsection{Homogenious Polarization Profiles}

The selection of the solvents was due to different molecular size and dipole moment. Acetone is the solvent with the highest dipole moment (2.88 Debye) while the alcohols all have nearly the same dipole moment ( $1.66-1.70$ Debye) but different in size. Hexane and $\mathrm{CCl}_{4}$ are nonpolar. 
After immersion of homogeneous poled films (field strength $\mathrm{E}=220 \mathrm{MV} / \mathrm{m}$, poling time $\mathrm{t}=5 \mathrm{~min}$ ) in acetone for $5.5 \mathrm{~min}$ the polarization was reduced by $40 \%$ (figure 1). The upper curve shows the remanent polarization before and the lower curve the remanent polarization after the contact with acetone. The immersion for some hours caused a weakening of polarization of $50 \%$.

The other solvents didn't show any meassurable influence on the polarization after 5 minutes of contact. Only the alcohols could reduce the polarization up to $15 \%$ but not until the films were immersed for some hours.

\subsubsection{Inhomogenious Polarization Profiles}

In this case the influence of polar and non polar solvents was much stronger. The poling field strength was $60 \mathrm{MV} / \mathrm{m}$ and the poling time 120 minutes. The immersion of samples in acetone caused a reduction of remanent polarization by $60-70 \%$ (figure 2 ). The upper curve shows the inhomogenious remanent polarization before and the lower curve the remanent polarization after the contact with acetone. Using alcohols the reduction was about $10-20 \%$ after an immersion of 5 minutes and about $40-50 \%$ after an immersion of some hours. Nonpolar solvents did not show an influence on the remanent polarization after some minutes. Surprisingly they had an influence on the remanent polarization after an immersion of several hours. Hexane caused a reduction by $20 \%$ and $\mathrm{CCl}_{4}$ up to $40 \%$.

The influence of solvents could be increased when the poling parameters of the samples were changed to $\mathrm{E}=80 \mathrm{MV} / \mathrm{m}$ and $\mathrm{t}=5 \mathrm{~min}$. For example the immersion in acetone for $5 \mathrm{~min}$ caused a reduction of remanent polarization by $80 \%$ from $\mathrm{P}=1.8 \mu \mathrm{C} / \mathrm{cm}^{2}$ to $\mathrm{P}=0.35 \mu \mathrm{C} / \mathrm{cm}^{2}$.

\subsection{Solvent Contact Before Poling}

By poling PVDF samples after the immersion in solvents we observed unusual polarization profiles. Poling parameters which normaly result in nearly homogenious polarization now caused strongly inhomogenious polarization profiles, sometimes with several maxima (mainly at polar solvents). Poling parameters which normaly result in inhomogenious polarization profiles under solvent influence lead to changed profiles in several variations. Figure 3 gives an example for a polarization profile after the immersion in acetone for some minutes. Even nonpolar solvents caused changed polarization profiles. Using hexane the maximum increased by $30 \%$ and the polarization was located at the side of the anode. The immersion in $\mathrm{CCl}_{4}$ resulted in an increased maximum of $10-20 \%$.

\section{DISCUSSION}

The fact that solvents can reduce the remanent polarization when poled PVDF films are immersed can be explained with the help of the charge trapping model [4] . This model suggests the trapping of charges which are injected into the film during poling with high electric fields. The charge traps arise at the surfaces of 
the crystallites in the form of Coulomb traps when the crystallite dipoles are

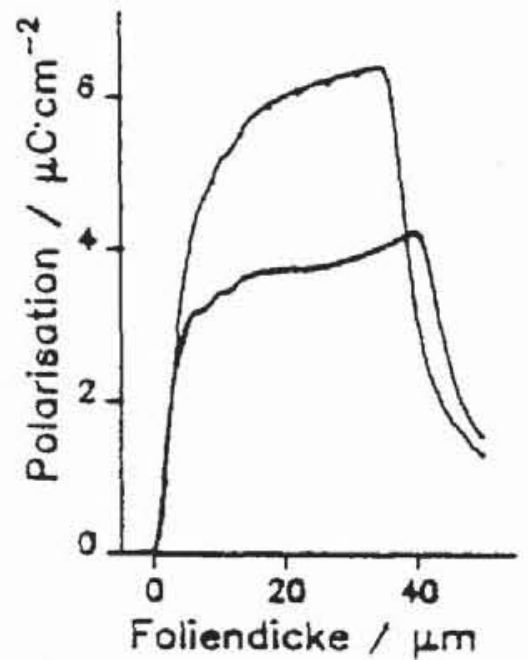

Figure 1: Polarization profile of a PVDF sample which was poled with $E=220 \mathrm{MV} / \mathrm{m}$ and $t=5 \mathrm{~min}$ and then immersed in acetone for 5 minutes. The upper curve shows the polarization before and the lower curve the polarization after the contact with acetone.



Figure 2: Polarization profile of a PVDF sample which was poled with $E=60 \mathrm{MV} / \mathrm{m}$ and $t=120 \mathrm{~min}$ and then immersed in acetone for 5 minutes. The upper curve shows the polarization before and the lower curve the polarization after the contact with acetone.

aligned in the applied field. After switching off the field the trapped charges compensate the aligned dipoles and stabilize them.

The presence of the solvent molecules in the film causes a swelling of the amorphous phase. Therefore charges can be freed which are mechanicly held in narrow holes at the transition from amorphous to crystalline phase. That may be the reason why nonpolar solvents lead to a small reduction of polarization.

On the other hand polar solvent molecules are able to accumulate to the trapped charges and decrease the trap binding Coulomb energie thereby thermal detrapping of weakly binded charges is possible. In fact, in the experiments polar solvents with higher dipole moment reduce the remanent polarization more than those with lower dipole moment.

The reduction of remanent polarization was larger at PVDF films poled with lower field strength than those poled with higher field strength. This is in accord with the charge trapping model predicting that higher fields in poling produce deeper traps.

The different polarization profiles of PVDF films which are poled after the immersion in a solvent can be discussed as follows:

Primarily all solvents cause higher conductivity of the PVDF. The largest change is observed when using acetone. There the conductivity increases by about four orders of magnitude [1]. Therefore at constant voltage the injection current increases in comparison to non-treated films. Consequently more 


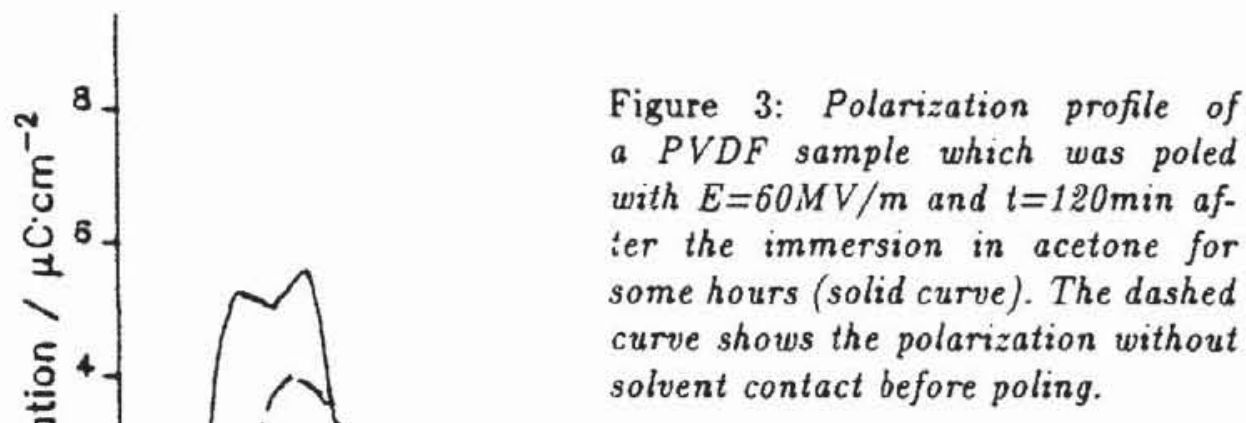

charges are available to be trapped.

At the beginning of poling the charges are trapped at the boundary of the polarization zone because of the high density of traps. When most of the dipoles are aligned the trap density doesn't increase any more. The existing traps are filled with charges and an increasing number of charges moves into the polarization zone. They are trapped until the polarization is stabilized with nearly homogenious charge distribution. The polarization current decreases to a minimum but does not vanish. Because of the present solvent the residual current is high enough to build up a great space charge and consequently an increasing field at a place where the polarization is still small or zero. New remanent polarization can arise. This explains the developement of the multiple peaks.

Because in PVDF the charge carriers are ions with different sizes the increase of conductivity is different for positive and negative charges. This leads to shifts of the position of the polarization maxima under the solvent influence.

\section{SUMMARY}

When poled PYDF films are immersed in different solvents the remanent polarization is reduced in dependence on the poling field, poling time, dipole moment of the solvent molecules, and the duration of solvent contact. One can obtain a stronger reduction of polarization by using smaller field strengths, longer poling time and immersion in the solvent, and high dipole moment of the solvent molecules. This behaviour can be explained with the interaction between the dipole moment of the solvent molecules and the charges which are bound in traps the depth of which depends on the poling field strength.

The long exposition to nonpolar solvents causes a small reduction of polarization after poling with low field but not with high field. 
The different polarization profiles which arise by poling PVDF films which contain solvents can be again explained with the help of the charge trapping model. The mobility of charges is increased by the solvent so that the remanent polarization grows faster and after its saturation more polarization peaks can arise besides the first peak.

\section{References}

[1] W. Burger,

"Ladungstransport in Polyvinylidenfluorid"

Diplomarbeit, 1.Phys.Instit., Universität Stuttgart, 1982

[2] W. Eisenmenger, M. Haardt, "Observation Of Charge Compensated Polarization Zones In Polyvinylidene (PVDF) Films By Piezoelectric Acoustic Step Wave Response"

Solid State Communications, Vol. 41, pp. 917-920, 1982.

[3] W. Eisenmenger, M. Haardt, K. Holdik, "Observation Of Charge Compensated Polarization Zones In Polyvinylidene Fluoride (PVDF) By Piezoelectrically Generated Pressure Step Wave (PPS) Response"

IEEE 1982 Annual Report Conf. Elect. Insul. and Dielect. Phen., pp. 5257,1982 .

[4] M. Haardt, W. Eisenmenger,

"High Resolution Technique For Meassuring Charge And Polarization Distributions In Dielectrics By Piezoelectrically Induced Pressure Step Waves (PPS)"

1982 Annual Report Conf. Elect. Insul. and Dielect. Phen., pp. 46-51, 1982.

[5] P. Fein, J. Kunz,

"Neue Konstruktionsmöglichkeiten mit Kunststoffen"

WEKA Fachverlage GmbH Kissing, Vol. 1, part 7, cap. 2.21, 1988.

[6] K. Holdik, W. Eisenmenger,

"Charge And Polarization Dynamics In Polymere Films"

Proc. 5th Int. IEEE Symp. Electrets, Piscataway, pp. 553-558, 1985. 